\title{
Free streamline flows with singularities
}

\author{
J. EGGERS $\dagger$ AND A. F. SMITH \\ School of Mathematics, University of Bristol, University Walk, Bristol BS8 1TW, UK
}

(Received 17 August 2009; revised 18 November 2009; accepted 20 November 2009)

We rederive and expand upon a method for finding solutions to the two-dimensional irrotational (inviscid) flow equations in the presence of a free surface, found by Hopkinson. This method allows the flow to be driven by placing singularities, like sources or vortices, in the interior of the flow domain. We then apply the method to find a number of novel solutions: separated flow driven by a source, vortices behind a plate and free-surface flow stirred by a double vortex. Free surfaces generically exhibit cusp singularities with a $2 / 3$ power index, similar to those found in very viscous flow.

\section{Introduction}

A streamline is called 'free' if the pressure along it is constant (Batchelor 1967). Such free streamlines arise in many physical problems in which fluid motion may be described in the potential flow approximation. One important example is the free surface between a fluid and a gas, if the effects of surface tension and hydrostatic pressure may be neglected. A second example arises in the context of separation from a solid body, which occurs if a body is placed in a flow at high Reynolds number. The shear layer between the outer flow and the stagnant wake region can be modelled to a reasonable approximation by a free streamline; see Smith (1986) for a discussion of the limitations of such an approach, relating in particular to the closure of the cavity. In two dimensions, powerful methods exist for finding exact solutions to free streamline flows, using complex mapping techniques.

However, almost all exact free streamline solutions known in the literature are limited by the way in which the flow may be driven. They involve either uniform streams that come in from infinity, or jets which come in from or escape to infinity. It would contribute much to the usefulness of the method if the flow could be driven from within the flow domain. In the context of inviscid flows, this is usually done by introducing singularities, such as sources or point vortices into the interior of the flow (Batchelor 1967). A few particular free streamline solutions with interior singularities are presented in the classical textbooks of Birkhoff \& Zarantonello (1957) and Gurevich (1966). The relation of interior singularities to small surface tension asymptotics has been explored in Chapman \& Vanden-Broeck (2002). The present paper is devoted to a general method which permits one to describe free streamline flows with any combination of internal singularities, for which the standard method fails. 
We consider steady solutions to the two-dimensional incompressible inviscid flow equations. If the flow is irrotational, the fluid velocity $\boldsymbol{u}=(u, v)$ satisfies

$$
\boldsymbol{u}=\nabla \phi, \quad \triangle \phi=0,
$$

where $\phi$ is the velocity potential. The pressure and the velocity are related by Bernoulli's equation (Landau \& Lifshitz 1984)

$$
\boldsymbol{u}^{2} / 2+p / \rho=\text { const. }
$$

According to (1.2), the fluid speed has to be constant along a free streamline. In this paper, we calculate the shape of free streamlines in the presence of singularities in the interior of the flow.

In two dimensions, the flow domain can be represented by a complex variable $z$, and the flow itself by the complex potential $w=\phi+\mathrm{i} \psi$. Here $\psi$ is the stream function, constant along streamlines. The flow field is determined by the derivative of the potential:

$$
\frac{\mathrm{d} w}{\mathrm{~d} z}=u-\mathrm{i} v \equiv q \mathrm{e}^{-\mathrm{i} \theta} .
$$

Evidently, $q$ is the speed of the fluid motion, and $\theta$ its direction. In the flow problems considered here, flow boundaries will be either solid surfaces or free streamlines. We will only consider the simplest case where the solids are flat plates, or at least composed of flat sections, with a constant angle between them. The problem to be dealt with is that the shape of the free surface is unknown.

A systematic way to rectify this problem was discovered by Kirchhoff (1869), and perfected by many others (Planck 1884; Love 1891). Namely, the variable

$$
\Omega=\ln \frac{\mathrm{d} z}{\mathrm{~d} w}=-\ln q+\mathrm{i} \theta
$$

maps the fluid domain onto a polygon: along a straight solid wall $\theta$ is constant, while along a free streamline $q$ is constant. According to the Schwarz-Christoffel theory (Carrier, Krook \& Pearson 1966), such a polygon can always be mapped onto the upper half of the complex plane (which we will always call the $\zeta$ domain), where the fluid motion is now represented. As shown in any standard textbook on fluid mechanics, the potential plane $w$ can also be represented in the same $\zeta$ plane, creating a link between $w$ and $\Omega$. This allows us to integrate the motion and to solve the two-dimensional flow equation.

The problem that presents itself in the presence of singularities is that the mapping between the physical domain $z$ and the upper half-plane $\zeta$ is no longer conformal. To fix this problem, two steps are required, as first described by Hopkinson (1898). First, singularities that will be present in the potential $w$ have to be subtracted, so as to keep the mapping $z(\zeta)$ conformal. Second, image singularities have to be placed in the lower half-plane, to make sure that both $w$ and $\Omega$ still map to the upper half-plane.

\section{The method}

The ultimate goal is to represent the fluid motion via a mapping $z(\zeta)$ in the upper half of the complex $\zeta$ plane, such that $z$ runs over the whole fluid domain. The crucial condition is that this mapping has no singularities in the upper half of the $\zeta$ plane; all singularities lie either on the real axis or in the lower half-plane, as detailed below. To find $z(\zeta)$, Hopkinson builds on a paper of Love (1891), which uses the differential 
expressions $\mathrm{d} w / \mathrm{d} \zeta$ and $\mathrm{d} \Omega / \mathrm{d} \zeta$ as starting points. Once they are found, one first finds $\Omega(\zeta)$ by integration. Then according to (1.4)

$$
\frac{\mathrm{d} z}{\mathrm{~d} \zeta}=\mathrm{e}^{\Omega} \frac{\mathrm{d} w}{\mathrm{~d} \zeta},
$$

from which $z(\zeta)$ is calculated by another integration.

It is clear that $w(\zeta)$ and $\Omega(\zeta)$ can be found by the usual process of SchwarzChristoffel mapping (Milne-Thompson 1962), as long as the fluid domain is bounded by free streamlines and piecewise straight solid walls. Namely, the boundaries of the fluid domain in the $w$ plane and the $\Omega$ plane are polygons, which can be mapped onto the upper $\zeta$ half-plane (Batchelor 1967). However, the beauty of the method lies in the fact that the method of finding the differential expressions is very constructive, based on the physical features of the problem. Moreover, this construction neatly separates the driving forces of the problem on one hand, and the geometry on the other. Thus the solution to any problem becomes a two-stage process:

(i) Driving forces encoded in the function $\mathrm{d} w / \mathrm{d} \zeta$. Along the boundaries of the fluid domain (both free and solid boundaries), the stream function is a constant. Thus the flow region in the $w$ domain consists of straight lines parallel to the real axis, and consequently $\mathrm{d} w / \mathrm{d} \zeta$ must be real on the real axis. The new feature of Hopkinson (1898) is that it considers flow driven by singularities from within the fluid domain. As a result, $\mathrm{d} w / \mathrm{d} z$ has poles of order $\alpha_{i}$ at certain discrete points $z_{i}$, which are mapped to corresponding points $\zeta_{i}$ in the $\zeta$ plane. Since $z(\zeta)$ is conformal, $\mathrm{d} w / \mathrm{d} \zeta$ has the same types of poles as presented physically. To ensure that in addition $\mathrm{d} w / \mathrm{d} \zeta$ be real for $\zeta$ real one has to add an image singularity at $\zeta_{i}^{*}$, which is the complex conjugate of the original pole.

In the classical problems usually solved in textbooks (Lamb 1932; Milne-Thompson 1962; Batchelor 1967), the flow is driven by jets or uniform streams coming from or escaping to infinity. Jets can be described by poles of order $\beta_{i}$, located at points $\zeta_{i}^{(r)} \in \mathbb{R}$ on the real $\zeta$-axis, and thus do not require an image singularity. A slightly different case is that of a uniform stream impinging on an obstacle. Such a stream can be viewed as a source located at $\zeta=\mathrm{i} \infty$. If an image is added at $\zeta=-\mathrm{i} \infty$, the corresponding potential becomes $\mathrm{d} w / \mathrm{d} \zeta=K \zeta$, a stagnation-point flow. In summary, $\mathrm{d} w / \mathrm{d} \zeta$ can be written as a superposition of $i=1 \ldots n$ poles of amplitude $A_{i}$ inside the flow, $m$ poles of amplitude $A_{i}^{(r)}$ on the real axis and a linear term:

$$
\frac{\mathrm{d} w}{\mathrm{~d} \zeta}=\sum_{i=1}^{n}\left(\frac{A_{i}}{\left(\zeta-\zeta_{i}\right)^{\alpha_{i}}}+\frac{A_{i}^{*}}{\left(\zeta-\zeta_{i}^{*}\right)^{\alpha_{i}}}\right)+\sum_{i=1}^{m} \frac{A_{i}^{(r)}}{\left(\zeta-\zeta_{i}^{(r)}\right)^{\beta_{i}}}+K \zeta .
$$

The first sum corresponds to any kind of singularity driving the flow from its interior. The second sum corresponds to jets coming from or escaping to infinity, while the last term describes a uniform stream.

(ii) Geometry encoded in $\mathrm{d} \Omega / \mathrm{d} \zeta$. The geometry of the problem is described by a succession of junctions between solid walls and free streamlines. According to (1.4), $\Omega$ has a piece parallel to the real axis on a solid wall, and a piece parallel to the imaginary axis on a free streamline. In the manner of Schwarz-Christoffel mapping (Carrier et al. 1966), the $90^{\circ}$ turns are achieved by placing singularities $s_{i}$ on the real axis of the $\zeta$ plane. Thus we obtain the following structure:

$$
\frac{\mathrm{d} \Omega}{\mathrm{d} \zeta}=\frac{\chi(\zeta)}{\sqrt{\prod_{i=1}^{l}\left(\zeta-s_{i}\right)}} \equiv \frac{\chi(\zeta)}{S(\zeta)}
$$


The function $\chi(\zeta)$ is needed to ensure that $z(\zeta)$ is conformal. An important constraint on $\chi$ is that it needs to be real on the real $\zeta$-axis, in order to achieve the correct Schwarz-Christoffel mapping.

To compute $\chi$, we observe that

$$
\Omega=\ln \frac{\mathrm{d} z}{\mathrm{~d} \zeta}-\ln \frac{\mathrm{d} w}{\mathrm{~d} \zeta},
$$

where $\mathrm{d} z / \mathrm{d} \zeta$ is conformal, i.e. free of singularities, in the upper half-plane. This means that $\Omega$, and thus $\mathrm{d} \Omega / \mathrm{d} \zeta$ must exhibit singularities at points where $\mathrm{d} w / \mathrm{d} \zeta$ has poles or zeros in the upper half-plane. In addition, there can be stagnation points of the flow on the solid boundaries, where $\mathrm{d} w / \mathrm{d} \zeta$ vanishes. At these points $\mathrm{d} z / \mathrm{d} \zeta$ remains regular as well, so $\Omega$ must become singular. The required singularity is determined from (2.4): let $(2.2)$ have a pole $(k<0)$ or zero $(k>0)$ at some point $\xi$, which may be real. Then

$$
\frac{\mathrm{d} w}{\mathrm{~d} \zeta} \propto(\zeta-\xi)^{k}, \quad \Rightarrow \frac{\mathrm{d} \Omega}{\mathrm{d} \zeta} \propto-\frac{k}{\zeta-\xi} .
$$

If $\xi_{i}$ is in the upper half-plane, $\mathrm{d} \Omega / \mathrm{d} \zeta$ also needs to have an image singularity, in order for it to be real on the real axis. Thus there are three different kinds of contributions to $\chi$, each resulting in a simple pole: first, there are $n$ poles at positions $\zeta_{i}$, coming from the poles of (2.2). Second, there are $n_{z}$ poles coming from zeros of (2.2), but $n_{z}$ may be zero; let us denote there position by $\xi_{i}$ and their order by $k_{i}$. Third, there are $n_{r}$ contributions from zeros of (2.2) on the real axis, but only if they come from a stretch corresponding to a solid surface; we denote their position by $\xi_{i}^{(r)}$ and their order by $k_{i}^{(r)}$. Again, their number may be zero. Thus the function $\chi$ is

$$
\chi=\sum_{i=1}^{n}\left(\frac{\alpha_{i} S\left(\zeta_{i}\right)}{\zeta-\zeta_{i}}+\frac{\alpha_{i} S^{*}\left(\zeta_{i}\right)}{\zeta-\zeta_{i}^{*}}\right)-\sum_{i=1}^{n_{z}}\left(\frac{k_{i} S\left(\xi_{i}\right)}{\zeta-\xi_{i}}+\frac{k_{i} S^{*}\left(\xi_{i}\right)}{\zeta-\xi_{i}^{*}}\right)-\sum_{i=1}^{n_{r}} \frac{k_{i}^{(r)} S\left(\xi_{i}^{(r)}\right)}{\zeta-\xi_{i}^{(r)}} .
$$

Another slight wrinkle to this construction, not described in Hopkinson (1898), is the case that some or all of the walls are not straight, but consist of several straight pieces, joined at an angle. This will result in $\mathrm{d} z / \mathrm{d} \zeta$ having corresponding singularities at $n_{c}$ places $\xi_{i}^{(c)}$ on the real axis. In the simplest case of right angles between the pieces (the generalization to arbitrary angles does not present any problem), these singularities will be of the form $1 / \sqrt{\left(\zeta-\xi_{i}^{(c)}\right)}$. In view of (2.4) this will result in $\Omega$ having corresponding logarithmic singularities $-\ln \left(\zeta-\xi_{i}^{(c)}\right) / 2$. Thus $\chi$ acquires another additive term

$$
\chi_{c}=-\frac{1}{2} \sum_{i=1}^{n_{c}} \frac{S\left(\xi_{i}^{(c)}\right)}{\zeta-\xi_{i}^{(c)}}
$$

\section{A classical solution: jet issuing from an orifice}

To illustrate the separation between driving forces and geometry introduced in the previous chapter, we start with a classical solution with no internal singularities. Our procedure will prove to be somewhat more constructive than the calculation presented in many textbooks (Lamb 1932; Milne-Thompson 1962; Batchelor 1967). The geometry is evident from figure 1, which shows the solution as obtained below. The 'driving force' in this problem is the jet going off to infinity. The corresponding 


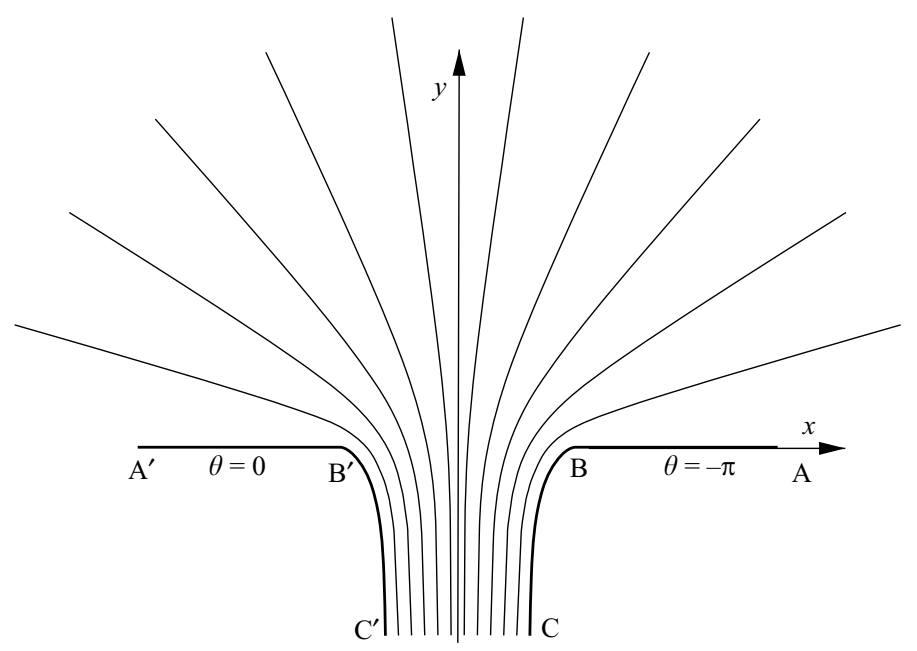

FIGURE 1. Fluid drains from a container through a slit to form a jet, which escapes to infinity at the lower part of the figure. The jet is bounded by the free surfaces between B and C, and B' and C', respectively. Streamlines are shown as well.

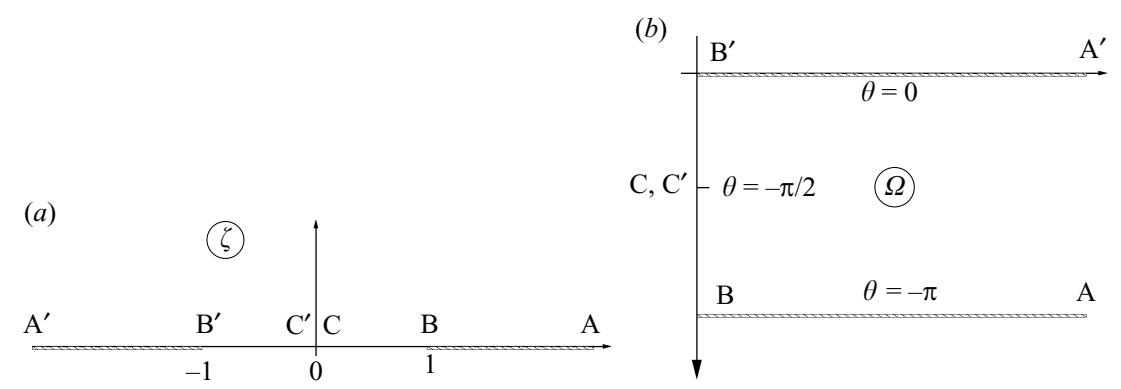

Figure 2. The $\zeta$ plane $(a)$, and the $\Omega$ plane $(b)$, corresponding to the jet problem of figure 1 .

singularity can be chosen at the origin of the $\zeta$ plane, so we have

$$
\frac{\mathrm{d} w}{\mathrm{~d} \zeta}=-\frac{1}{\zeta}
$$

and the $\zeta$ plane looks as shown in figure $2(a)$. In choosing a characteristic time scale for the problem, we have normalized the prefactor in (3.1) to unity. This leaves all geometrical properties unaffected. We have chosen a minus sign since the jet behaves like a source, and fluid leaves the system towards infinity. A picture of the streamlines is shown in figure 1; thus the boundaries in the $\Omega$ plane look as shown in figure $2(b)$.

In the $\zeta$ plane, we have chosen the boundaries between solid-liquid-solid at $\zeta= \pm 1$. This means that $\chi=1$, since there are no singularities of (3.1) in the upper half-plane nor zeros on the real axis, and

$$
\frac{\mathrm{d} \Omega}{\mathrm{d} \zeta}=\frac{1}{\sqrt{\zeta^{2}-1}} .
$$

For $|\zeta|>1, \mathrm{~d} \Omega / \mathrm{d} \zeta$ is real and positive, and for $|\zeta|<1$ turns purely imaginary. Integrating (3.2) we find

$$
\Omega=\operatorname{arccosh} \zeta-\mathrm{i} \pi=\ln \left(\zeta+\sqrt{\zeta^{2}-1}\right)-\mathrm{i} \pi
$$


where the constant of integration has been chosen to match figure 2. Thus

$$
\frac{\mathrm{d} z}{\mathrm{~d} \zeta}=\mathrm{e}^{\Omega} \frac{\mathrm{d} w}{\mathrm{~d} \zeta}=\left(\zeta+\sqrt{\zeta^{2}-1}\right) \frac{1}{\zeta}=1+\mathrm{i} \frac{\sqrt{1-\zeta^{2}}}{\zeta}
$$

and integral of this expression is

$$
z=\zeta+\mathrm{i}\left[\sqrt{1-\zeta^{2}}+\ln \left(\frac{1+\sqrt{1-\zeta^{2}}}{\zeta}\right)\right]+z_{0}
$$

The jet corresponds to $\zeta=0$, where the real part of (3.5) suffers a jump of $\pi$. Thus $z_{0}=\pi / 2$ to place the jet at the line of symmetry, and

$$
\begin{aligned}
& x=\zeta+\pi / 2, \\
& y=\sqrt{1-\zeta^{2}}+\ln \left(\frac{1+\sqrt{1-\zeta^{2}}}{\zeta}\right),
\end{aligned}
$$

for $0 \leqslant \zeta \leqslant 1$ is the equation for the shape of the jet, shown as the heavy line between $\mathrm{B}$ and $\mathrm{C}$ in figure 1. The flow lines are described by $\zeta=A \mathrm{e}^{\mathrm{i} \theta}$ in the $\zeta$ plane, where $\theta$ is a constant angle. If one inserts this expression into (3.5), $z$ will trace out the flow lines as $A$ is varied from 0 to $\infty$.

\section{A point source next to a wall}

Inviscid flow problems driven by sources and sinks placed inside the fluid have been considered in many previous papers (Vanden-Broeck \& Keller 1987; Forbes \& Hocking 1990; Mekias \& Vanden-Broeck 1991), but they are generally treated numerically using boundary integral methods. In some very special cases, exact solutions exist (Sautreaux 1901; Craya 1949). We consider exact solutions to flow problems driven by a (two-dimensional) point source of fluid placed at a distance $H$ from a (solid) wall. If there is no separation (the fluid spreads along the wall to infinity), the problem is solved classically by placing an image source on the other side of the wall (cf. Milne-Thompson 1962, p. 210). We now present a different solution to the problem in which the flow separates from the wall at a position A (cf. figure 3 ). The shape of the free streamline, which marks the boundary between the source flow and the surrounding fluid, needs to be determined as part of the solution. In the $\zeta$ plane, the flow is the same as in the aforementioned unseparated problem, where we choose the position of the source $\zeta=\mathrm{i} \gamma$, and hence

$$
\frac{\mathrm{d} w}{\mathrm{~d} \zeta}=\frac{1}{\zeta-\mathrm{i} \gamma}+\frac{1}{\zeta+\mathrm{i} \gamma}=\frac{2 \zeta}{\zeta^{2}+\gamma^{2}}
$$

so that $\mathrm{d} w / \mathrm{d} \zeta$ is real for real $\zeta$, as required. Now the $\zeta$ plane looks as shown in figure 4 , where the half width of the unseparated region is chosen as unity.

This means the function $\Omega$ must satisfy

$$
\frac{\mathrm{d} \Omega}{\mathrm{d} \zeta}=\frac{\chi(\zeta)}{\sqrt{1-\zeta^{2}}}
$$

where $\chi$ is real on the real axis. As explained in $\S 2, \chi$ is the sum of contributions coming from poles and zeros of $\mathrm{d} w / \mathrm{d} \zeta$. Namely, (4.1) has a simple pole for $\zeta=\mathrm{i} \gamma$ 


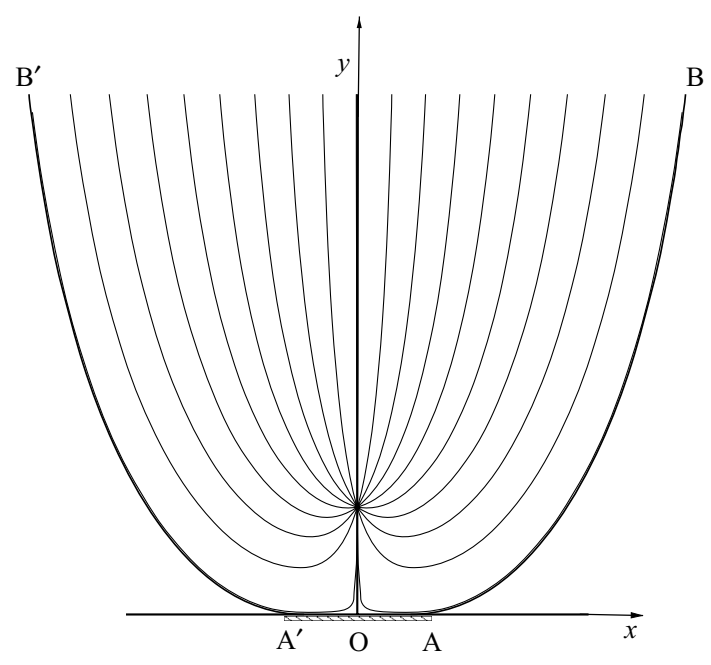

FiguRE 3. A two-dimensional source placed at a distance $H$ from a plane wall. At a distance $d$ from the line of symmetry (marked by symbols A and $\mathrm{A}^{\prime}$ ), the flow separates from the wall. Outside, the fluid is taken to be stagnant.

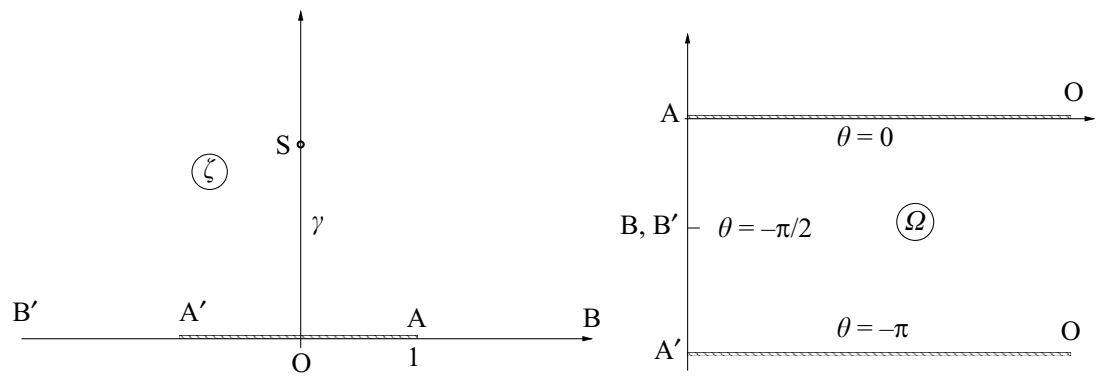

Figure 4. The $\zeta$ plane $(a)$, and the $\Omega$ plane $(b)$, corresponding to figure 3 . A source, marked $\mathrm{S}$, is placed at a distance $\gamma$ from the wall in the $\zeta$ plane.

and has a zero for $\zeta=0$, which is on the solid wall. Using (2.6), it follows that

$$
\chi=-\frac{1}{\zeta}+\frac{\sqrt{1+\gamma^{2}}}{\zeta-\mathrm{i} \gamma}+\frac{\sqrt{1+\gamma^{2}}}{\zeta+\mathrm{i} \gamma}=\frac{2 \zeta \sqrt{1+\gamma^{2}}}{\zeta^{2}+\gamma^{2}}-\frac{1}{\zeta}
$$

which includes an image singularity at $\zeta=-\mathrm{i} \gamma$ to ensure that $\chi$ is real for real $\zeta$.

The expression for $\mathrm{d} \Omega / \mathrm{d} \zeta$ corresponding to (4.3) can be integrated conveniently term by term. It is useful to use the transformation $\zeta=\sin \varphi, \sqrt{1-\zeta^{2}}=\cos \varphi$ :

$$
\Omega_{0} \equiv-\int \frac{\mathrm{d} \zeta}{\zeta \sqrt{1-\zeta^{2}}}=-\int \frac{\mathrm{d} \varphi}{\sin \varphi}=\ln \left(\frac{1+\cos \varphi}{\sin \varphi}\right)
$$

and

$$
\Omega_{\gamma} \equiv \int \frac{2 \zeta \sqrt{1+\gamma^{2}} \mathrm{~d} \zeta}{\left(\zeta^{2}+\gamma^{2}\right) \sqrt{1-\zeta^{2}}}=\int \frac{2 \sin \varphi \cosh \alpha \mathrm{d} \varphi}{\cosh ^{2} \alpha-\cos ^{2} \varphi}=\ln \left(\frac{\cosh \alpha-\cos \varphi}{\cosh \alpha+\cos \varphi}\right) .
$$




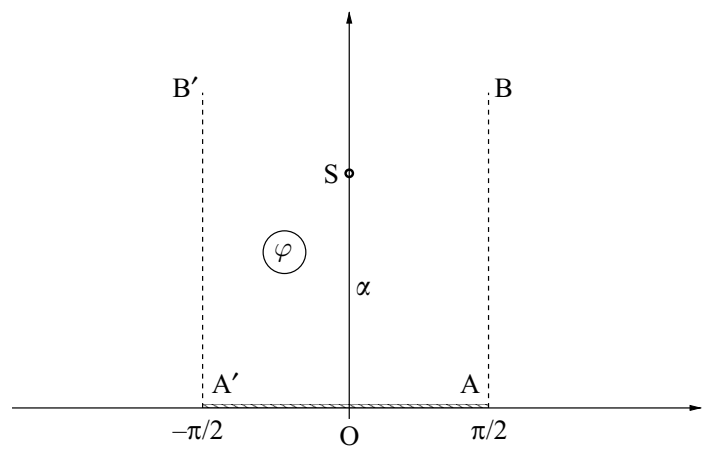

Figure 5. The $\varphi$ plane corresponding to figure 3, coming from the transformation $\zeta=\sin \varphi$ of the $\zeta$ plane (cf. figure 4). In the $\varphi$ plane, the source is at a distance $\alpha$ from the wall.

where $\gamma=\cosh \alpha$ (see figure 5). Thus the transformation to the physical plane obeys

$$
\frac{\mathrm{d} z}{\mathrm{~d} \varphi}=\mathrm{e}^{\Omega_{0}+\Omega_{\gamma}} \frac{\mathrm{d} w}{\mathrm{~d} \zeta} \cos \varphi=2 \frac{(1+\cos \varphi) \cos \varphi}{(\cos \varphi+\cosh \alpha)^{2}} .
$$

The integral of this expression can be brought into the relatively simple form

$$
\begin{array}{r}
I(\varphi, \beta) \equiv \int \frac{(1+\cos \varphi) \cos \varphi}{(\cos \varphi+\beta)^{2}}=-2 \frac{\beta^{2}+\beta-1}{(\beta+1)^{3 / 2}(\beta-1)^{1 / 2}} \arctan \left(\sqrt{\frac{\beta-1}{\beta+1}} t\right) \\
-\frac{2 \beta t}{\left(\beta^{2}-1\right) t^{2}+(\beta+1)^{2}}+\varphi,
\end{array}
$$

where $\beta=\cosh \alpha=\sqrt{1+\gamma^{2}}$ and where we have put $t=\tan (\varphi / 2)$ for brevity. Now the mapping that describes the geometry of the free streamlines is

$$
z=2 I(\varphi, \beta)
$$

As seen in figure 5 , the free streamlines are described by $\varphi$ varying along the dashed lines; $\varphi=\pi / 2+\mathrm{i} \psi$, where $\psi$ varies between 0 and $\infty$, is a parametrization of the free streamline. Let us describe briefly how this transformation corresponds to variables in physical space. If $H$ is the distance of the source from the wall, and $d$ the distance of the point of separation $A$ from the line of symmetry, one obtains

$$
d=\frac{\mathrm{i} I(\pi / 2, \beta)}{I(\mathrm{i} \alpha, \beta)} H .
$$

Further, the flow between the free streamlines must be uniform far away from the wall, so $Q / V$ is the asymptotic distance between the free streamlines, where $Q$ is the volume flux of the flow, and $V$ the speed on the free streamline. For $\psi \rightarrow \infty$ one finds $t=\mathrm{i}$, so $I(\pi / 2+\psi, \beta) \rightarrow \pi / 2$. Thus one finds

$$
\frac{Q}{V H}=\frac{\mathrm{i} \pi}{I(\mathrm{i} \alpha, \beta)} \text {. }
$$

To trace the streamlines inside the flow, one can take the following steps: first, one has to find the lines along which the stream function is constant in the $\zeta$ plane, i.e. lines of constant $\operatorname{Im}\{w\}$. Unfortunately, these curves can usually not be parametrized in closed form. Second, the curves have to be transformed into the $z$ plane using $z(\zeta)$. To avoid this cumbersome procedure, we formulate a system of differential equations 


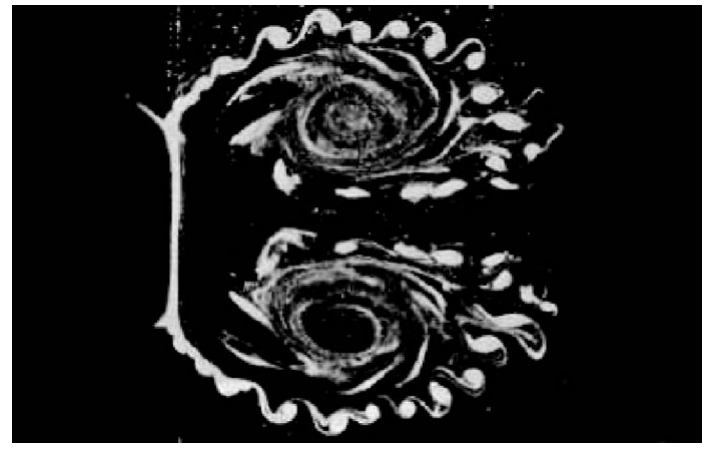

FIGURE 6. The flow around an impulsively started flat plate (Taneda 1987), at a Reynolds number of 3857.

whose solution traces the streamlines as function of some parameter $s$. To this end we require that $\mathrm{d} \psi / \mathrm{d} s=0$ and $\mathrm{d} \phi / \mathrm{d} s=1$, leading to

$$
\frac{\mathrm{d} \zeta_{r}}{\mathrm{~d} s}=\operatorname{Re}\left\{\frac{\mathrm{d} w}{\mathrm{~d} \zeta}\right\} /\left|\frac{\mathrm{d} w}{\mathrm{~d} \zeta}\right|^{2}, \quad \frac{\mathrm{d} \zeta_{i}}{\mathrm{~d} s}=-\operatorname{Im}\left\{\frac{\mathrm{d} w}{\mathrm{~d} \zeta}\right\} /\left|\frac{\mathrm{d} w}{\mathrm{~d} \zeta}\right|^{2} .
$$

To find the parameter form $(x(s), y(s))$ of a free streamline, one also has to solve the equations

$$
\frac{\mathrm{d} x}{\mathrm{~d} s}=\operatorname{Re}\left\{\frac{\mathrm{d} z}{\mathrm{~d} \zeta} \frac{\mathrm{d} \zeta}{\mathrm{d} s}\right\}, \quad \frac{\mathrm{d} y}{\mathrm{~d} s}=\operatorname{Im}\left\{\frac{\mathrm{d} z}{\mathrm{~d} \zeta} \frac{\mathrm{d} \zeta}{\mathrm{d} s}\right\}
$$

The flow lines shown in figure 3 have been obtained by solving the four equations (4.11), (4.12) simultaneously, with initial conditions in all directions around the source.

\section{Two vortices behind a flat plate}

We now model two vortices such as they appear behind a bluff body, for example, when a plate (Hudson \& Dennis 1985) or a sphere (Elcrat, Fornberg \& Miller 2001) is placed in a uniform stream. However, since we have not attempted to describe the outer flow past the body, our solution is a better model for the case that the wake flow is surrounded by stagnant fluid. Such a flow is created by setting the plate in sudden motion, and then stopping it again (see figure 6). Vorticity is introduced into the flow by separation from the edges of the plate (Saffman 1992), and two counter-rotating vortices form, which appear to have a well-defined core position. A shear layer forms, which is unstable to the Kelvin-Helmholtz instability, and results in a sequence of small-scale vortices. Our model approximates the shear layer as a jump in the velocity, and of course does not include any of the instabilities (see figure $7 b$ ).

The singularity of a single vortex behaves like $w \propto-\mathrm{i} \ln z$. Thus the contribution of a vortex of strength $m$ at $a=\alpha+\mathrm{i} \beta$ in the $\zeta$ plane, including the image vortex at $a^{*}$, is

$$
\frac{\mathrm{d} w}{\mathrm{~d} \zeta}=-\mathrm{i} m\left[\frac{1}{\zeta-a}-\frac{1}{\zeta-a^{*}}\right]=\frac{2 m \beta}{(\zeta-\alpha)^{2}+\beta^{2}}
$$




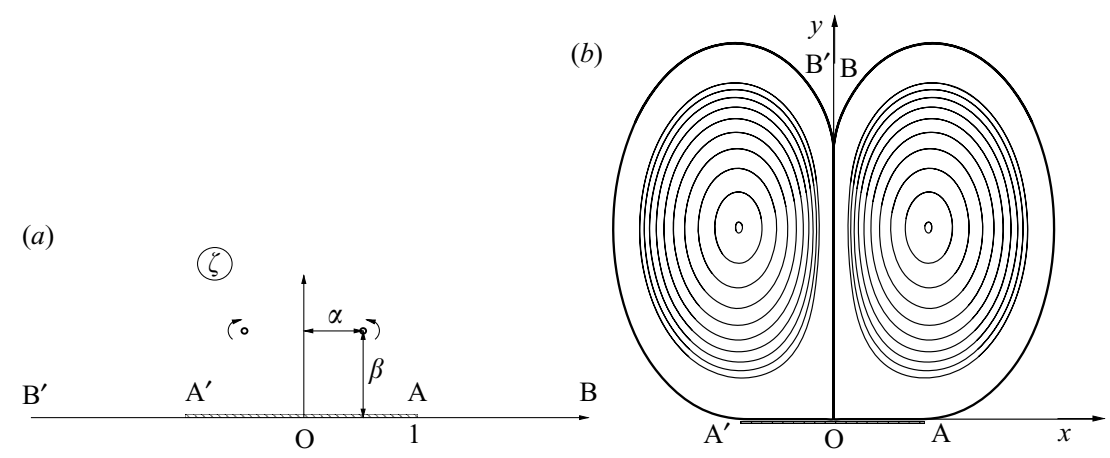

Figure 7. (a) The $\zeta$ plane for two vortices behind a plate. The sense of rotation of the vortices is indicated by arrows. $(b)$ The realization in physical space; parameters are $\alpha=1.85$ and $\beta=0.9$. The heavy line is the shear layer, which bounds the recirculation zone.

Then the contribution of two counter-rotating vortices, placed symmetrically about the imaginary axis, is

$$
\frac{\mathrm{d} w}{\mathrm{~d} \zeta}=\frac{1}{(\zeta-\alpha)^{2}+\beta^{2}}-\frac{1}{(\zeta+\alpha)^{2}+\beta^{2}}
$$

choosing $m=1 /(2 \beta)$. It is clear that (5.2) also has a stagnation point at $\zeta=0$.

The function $\mathrm{d} \Omega / \mathrm{d} \zeta$ retains the same structure as (4.2), and $\chi$ is determined by the poles and zeros of (5.2) according to (2.6). Let us focus first on the pole contribution $\Omega_{a}$ of the vortex at $a$, which gives

$$
\chi_{a}=\frac{\sqrt{1-a^{2}}}{\zeta-a}+\frac{\sqrt{1-\left(a^{*}\right)^{2}}}{\zeta+a^{*}} .
$$

Integrating the corresponding expression (4.2), we find

$$
\Omega_{a}=\ln \frac{\left(\tan (\varphi / 2)-c_{-}\right)\left(\tan (\varphi / 2)-c_{-}^{*}\right)}{\left(\tan (\varphi / 2)-c_{+}\right)\left(\tan (\varphi / 2)-c_{+}^{*}\right)}, \quad c_{ \pm}=\frac{1 \pm \sqrt{1-a^{2}}}{a},
$$

where $\sin \zeta=\varphi$ as usual. Thus the contribution from two counter-rotating vortices, placed at $a_{1}=\alpha+\mathrm{i} \beta$ and $a_{2}=-\alpha+\mathrm{i} \beta$, and from the stagnation point at $\zeta=0,(4.4)$, gives

$$
\frac{\mathrm{d} z}{\mathrm{~d} \varphi}=\mathrm{e}^{\Omega_{a_{1}}+\Omega_{a_{2}}+\Omega_{0}} \frac{\mathrm{d} w}{\mathrm{~d} \zeta} \cos \varphi
$$

The resulting flow is shown in figure 7(b), for a particular choice of the position of the two vortices in the $\zeta$ plane. The position in real space must be found by integrating (5.5). The flow lines are drawn using the procedure described in the previous section. The free streamlines are found by seeding an initial condition very close to the solid plate.

\section{A stirred teacup}

We now address the deformation of a free liquid-gas interface by a strong flow in the interior of the fluid. This means surface tension and gravity are negligible compared to fluid inertia. Following earlier work in an analogous viscous flow problem (Jeong \& Moffatt 1992), we drive the flow by a double vortex. A very similar inviscid freesurface problem was studied theoretically and numerically by Verga (2004), however 
(a)

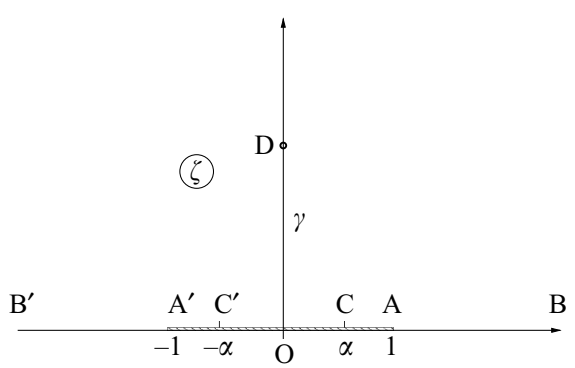

(b)

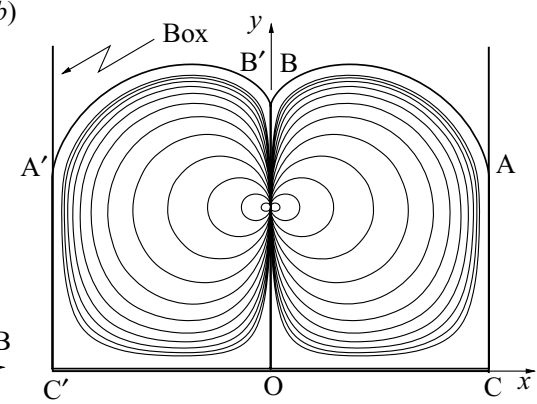

Figure 8. (a) The $\zeta$ plane for the physical situation shown in $(b)$, which is a vortex doublet, marked D, placed inside a box filled with fluid at a distance $\gamma$ form the bottom. A cusp forms on the free surface. Parameters for the situation shown are $\gamma=1$ and $\alpha=1 / 2$.

without giving the exact solution reported below. In the $\zeta$ representation, we place the double vortex at $\zeta=\mathrm{i} \gamma$, and its singularity is $w \propto \mathrm{i} /(\zeta-\mathrm{i} \gamma)$. Thus, including the image singularity, $\mathrm{d} w / \mathrm{d} \zeta$ becomes

$$
\frac{\mathrm{d} w}{\mathrm{~d} \zeta}=\frac{\zeta}{\left(\zeta^{2}-\gamma^{2}\right)^{2}}
$$

where we have normalized the strength of the vortex doublet as usual. As is clear from the geometry of the problem (see also figure 8), the form of $\mathrm{d} \Omega / \mathrm{d} \zeta$ is given by (4.2), as in the two previous problems. According to (2.7), we first find the poles and zeros of (6.1) in the upper half-plane or the real axis. There is a simple zero at $\zeta=0$, and a double pole at $\zeta=\mathrm{i} \gamma$, to which an image contribution has to be added. In addition, to describe the form of the box, there are singularities at $s_{1 / 2}= \pm \alpha$, so (2.7) becomes

$$
\chi=\frac{4 \zeta \sqrt{1+\gamma^{2}}}{\zeta^{2}+\gamma^{2}}-\frac{1}{\zeta}+\frac{\zeta \sqrt{1-\alpha^{2}}}{\zeta^{2}-\alpha^{2}}
$$

The contribution coming from the last term of (6.2) is

$$
\Omega_{\alpha} \equiv \int \frac{\zeta \sqrt{1-\alpha^{2}} \mathrm{~d} \zeta}{\left(\zeta^{2}-\alpha^{2}\right) \sqrt{1-\zeta^{2}}}=\int \frac{\sin \varphi \sqrt{1-\alpha^{2}} \mathrm{~d} \varphi}{\cos ^{2} \varphi-1+\alpha^{2}}=\ln \left[\left(\frac{\cos \varphi+\sqrt{1-\alpha^{2}}}{\cos \varphi-\sqrt{1-\alpha^{2}}}\right)^{1 / 2}\right]
$$

which gives

$$
\frac{\mathrm{d} z}{\mathrm{~d} \varphi}=\mathrm{e}^{\Omega_{0}+2 \Omega_{\gamma}+\Omega_{\alpha}} \frac{\mathrm{d} w}{\mathrm{~d} \zeta} \cos \varphi=\frac{(1+\cos \varphi) \cos \varphi}{\left(\cos \varphi+\sqrt{1+\gamma^{2}}\right)^{4}}\left(\frac{\cos \varphi+\sqrt{1-\alpha^{2}}}{\cos \varphi-\sqrt{1-\alpha^{2}}}\right)^{1 / 2},
$$

which can be integrated in terms of elliptic integrals. However, in practise we found it more useful to solve the system (4.11), (4.12) numerically. The width $w$ and height $h$ of the box is given by integrating $\varphi$ from 0 to $\pi / 2$ :

$$
(w, i h)=\int_{0}^{\pi / 2} \frac{\mathrm{d} z}{\mathrm{~d} \varphi} \mathrm{d} \varphi
$$

As seen in figure $8(b)$, there is a cusp singularity that forms on the free surface, at least in the limit of vanishing surface tension. In the $\zeta$ plane, the boundary between 
solid and free surface is at $\zeta=1$, and the cusp occurs for $\zeta \rightarrow \infty$. Thus if one puts $\varphi=\pi / 2+\mathrm{i} \chi$, the cusp is described by the limiting behaviour of the mapping for $\chi \rightarrow \infty$. Using $\sin \varphi=\cosh \chi$ and $\cos \varphi=-\mathrm{i} \sinh \chi$, it is easy to verify that the square root factor in (6.4) goes to unity in the limit. From the remaining expressions one obtains

$$
\frac{\mathrm{d} x}{\mathrm{~d} \chi}=-\operatorname{Im}\left\{\frac{\mathrm{d} z}{\mathrm{~d} \varphi}\right\} \approx 16 \sqrt{1+\gamma^{2}} \mathrm{e}^{-3 \chi}, \quad \frac{\mathrm{d} y}{\mathrm{~d} \chi}=\operatorname{Re}\left\{\frac{\mathrm{d} z}{\mathrm{~d} \varphi}\right\} \approx 4 \mathrm{e}^{-2 \chi}
$$

In other words, the limiting shape of the cusp is

$$
x=x_{0}-\frac{16}{3} \sqrt{1+\gamma^{2}} \mathrm{e}^{-3 \chi}, \quad y=y_{0}-2 \mathrm{e}^{-2 \chi},
$$

which implies that the cusp is of the generic form $x \propto y^{3 / 2}$. Cusps have previously been observed in a number of related two-dimensional potential flow problems, for example, in drop driven by a combination of a single and a double vortex (Hopkinson 1898), or by a source (Sautreaux 1901; Craya 1949; Vanden-Broeck \& Keller 1987). In each case, we have confirmed that the local structure is the same $2 / 3$ cusp. This is to be expected, since the structure of a singularity will be independent of the large-scale flow far from it. However, it is remarkable that the same type of cusp is also found in the very different limit of very viscous fluids (Jeong \& Moffatt 1992). The ubiquity of cusps in free-surface flows remains an open problem, and is discussed in more detail in Eggers \& Fontelos (2009).

\section{Discussion}

In this paper, we hope to have demonstrated that Hopkinson's method greatly expands the repertoire of physically realistic free streamline flows, for which an exact solution can be found. To this end we have reformulated the method in an algorithmic form, which neatly separates the physical ingredients to a given problem. One must specify both the forces that drive the flow, and one must specify the geometry, consisting of solid walls and free surfaces. Once these two parts of the problem are given, the solution can be found as prescribed in $\S 2$. In addition, we also extended Hopkinson's result to the case that the solid has corners.

We then use the method to construct three new free streamline flows, all of which correspond to physically realizable experimental set-ups. Of particular current interest is the example of $\S 6$, which reveals that a strongly stirred free-surface flow will form cusps. In a three-dimensional setting, they correspond to line-like 'scars' on the free surface. One example illustrating the formation of scars is the turbulent flow shown in figure 9, whose free surface is covered by a network of such lines. This network appears to organize the overall structure of the free surface. As illustrated in Brocchini \& Peregrine (2001), there is an upwelling of the flow in between scars, while there is downwelling at the position of the scar.

In addition, scars may be the locus where air is entrained into the fluid (Brocchini \& Peregrine 2001), as was demonstrated previously for the case of viscous flow (Eggers 2001; Lorenceau, Quéré \& Eggers 2004). However, to achieve a proper description of this process, the outer phase has of course to be included. Near the locus of entrainment, surface tension and viscosity are likely to be important as well. The downwelling near the cusp implies that there is flow separation taking place from the free surface, whose properties have been investigated recently (Sébilleau, 


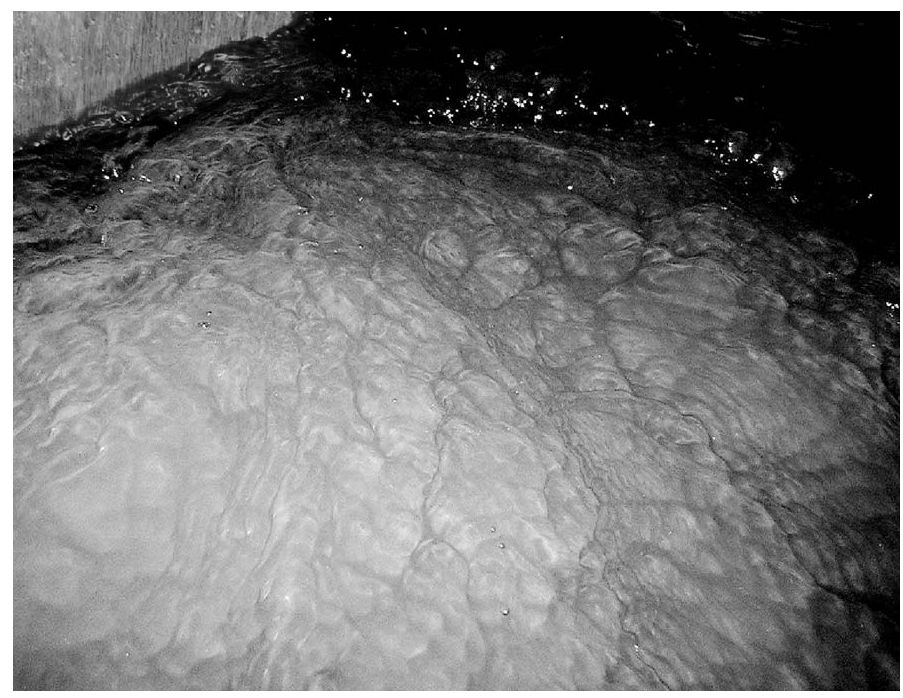

FIGURE 9. The free surface of a turbulent flow. On the surface a network of grooves or 'scars' is visible; unpublished photograph by Howell Peregrine.

Limat \& Eggers 2009). In particular, the viscous boundary conditions are necessary for a proper local description of flow separation.

The solution of a separated flow generated by a source near a wall was found originally and shown to one of us (J. Eggers) by the late Howell Peregrine. The derivation had however been lost, and was therefore proposed as a Master's project at the University of Bristol's Mathematics department.

\section{REFERENCES}

Batchelor, G. K. 1967 An Introduction to Fluid Dynamics. Cambridge University Press.

Birkhoff, G. \& Zarantonello, E. H. 1957 Jets, Wakes and Cavities. Academic.

Brocchini, M. \& Peregrine, D. H. 2001 The dynamics of strong turbulence at free surfaces. Part 1. Description. J. Fluid Mech. 449, 225-254.

Carrier, G. F., Krook, M. \& Pearson, C. E. 1966 Functions of a Complex Variable. McGraw-Hill.

Chapman, S. J. \& Vanden-BroecK, J.-M. 2002 Exponential asymptotics and capillary waves. SIAM J. Appl. Math. 62, 1872-1898.

CrayA, A. 1949 Theoretical research on the flow of nonhomogeneous fluids. La Houille Blanche 4, $44-55$.

EgGers, J. 2001 Air entrainment through free-surface cusps. Phys. Rev. Lett. 86, 4290.

EgGers, J. \& Fontelos, M. 2009 Cusps in interfacial problems. http://arxiv:math-ph/0910.3499.

Elcrat, A. R., Fornberg, B. \& Miller, K. G. 2001 Some steady axisymmetric vortex flows past a sphere. J. Fluid Mech. 433, 315-328.

Forbes, L. K. \& Hocking, G. C. 1990 Flow caused by a point sink in a fluid having a free surface. J. Aust. Math. Soc. B 32, 231-249.

Gurevich, M. I. 1966 Theory of Jets in an Ideal Fluid. Pergamon.

Hopkinson, B. 1898 Discontinuous fluid motions involving sources and vortices. Proc. Lond. Math. Soc. 29, 142-164.

Hudson, J. D. \& Dennis, S. C. R. 1985 The flow of a viscous incompressible fluid past a normal flat plate at low and intermediate Reynolds numbers: the wake. J. Fluid Mech. 160, 369-383.

JeOng, J.-T. \& Moffatt, H. K. 1992 Free-surface cusps associated with a flow at low Reynolds numbers. J. Fluid Mech. 241, 1-22. 
Kirchноf, G. 1869 Zur Theorie freier Flüssigkeitsstrahlen. J. reine angew. Math. 70, 289.

Lamb, H. 1932 Hydrodynamics, 6th edn. Cambridge University Press.

Landau, L. D. \& Lifshitz, E. M. 1984 Fluid Mechanics. Pergamon.

LORENCEAU, E., Quéré, D. \& EGGERs, J. 2004 Air entrainment by a viscous jet plunging into a bath. Phys. Rev. Lett. 93, 254501.

Love, A. E. H. 1891 On the theory of discontinuous fluid motion in two dimensions. Proc. Camb. Phil. Soc. 7, 175-201.

Mekias, H. \& VANDEN-Broeck, J.-M. 1991 Subcritical flow with a stagnation point due to a source beneath a free surface. Phys. Fluids A 3, 2652-2658.

Milne-Thompson, L. M. 1962 Theoretical Hydrodynamics, 4th edn. Macmillan \& Co.

Planck, M. 1884 Zur Theorie der Flüssigkeitsstrahlen. Wied. Ann. 21, 499-509.

Saffman, P. G. 1992 Vortex Dynamics. Cambridge University Press.

Sautreaux, C. 1901 Mouvement d'un liquide parfait soumis à la pesanteur. Détermination des lignes de courant. J. Math. Pures Appl. 7, 125-159.

Sébilleau, J., Limat, L. \& EgGers, J. 2009 Flow separation from a stationary meniscus. J. Fluid Mech. 633, 137.

Sмiтн, F. T. 1986 Steady and unsteady boundary layer separation. Annu. Rev. Fluid Mech. 18, 197-220.

TANEDA, S. 1987 Irregular flows. Sadhana 10, 349-375.

Vanden-Broeck, J.-M. \& Keller, J. B. 1987 Free surface flow due to a sink. J. Fluid Mech. 175, $109-117$.

Verga, A. 2004 Singularity formation in vortex sheets and interfaces. Nonlinear Phenom. Complex Syst. 9, 361-388. 